\title{
IRAD experience on surgical type A acute dissection patients: results and predictors of mortality
}

\author{
Paolo Berretta ${ }^{1}$, Himanshu J. Patel ${ }^{2}$, Thomas G. Gleason ${ }^{3}$, Thoralf M. Sundt ${ }^{4}$, Truls Myrmel ${ }^{5}$, \\ Nimesh Desai ${ }^{6}$, Amit Korach ${ }^{7}$, Antonello Panza ${ }^{8}$, Joe Bavaria ${ }^{6}$, Ali Khoynezhad ${ }^{9}$, Elise Woznicki ${ }^{10}$, \\ Dan Montgomery $^{10}$, Eric M. Isselbacher ${ }^{4}$, Roberto Di Bartolomeo ${ }^{11}$, Rossella Fattori ${ }^{12}$, Christoph A. \\ Nienaber $^{13}$, Kim A. Eagle ${ }^{10}$, Santi Trimarchi ${ }^{14}$, Marco Di Eusanio ${ }^{1}$ \\ ${ }^{1}$ Division of Cardiac Surgery, G. Mazzini Hospital, Teramo, Italy; ${ }^{2}$ Cardiac Surgery, University of Michigan Health System, Ann Arbor, USA; \\ ${ }^{3}$ Division of Cardiothoracic Surgery, University of Pittsburgh, Pittsburgh, USA; ${ }^{4}$ Thoracic Aortic Center, Massachusetts General Hospital, Boston, \\ USA; ${ }^{5}$ Department of Thoracic and Cardiovascular Surgery, Tromsø University Hospital, Tromsø, Norway; ${ }^{6}$ Division of Cardiovascular Surgery, \\ University of Pennsylvania School of Medicine, Philadelphia, USA; ${ }^{7}$ Department of Cardiothoracic Surgery, Hadassah Hebrew University Medical \\ Center, Jerusalem, Israel; ${ }^{8}$ Department of Cardiology and Cardiac Surgery, University Hospital 'San Giovanni di Dio e Ruggi d'Aragona', Salerno, \\ Italy; ${ }^{9}$ Division of Cardiothoracic Surgery, Cedars-Sinai Medical Center, Los Angeles, USA; ${ }^{10}$ Cardiovascular Center, University of Michigan Health \\ System, Ann Arbor, USA; ${ }^{11}$ Department of Cardiac Surgery, S.Orsola-Malpighi Hospital, University of Bologna, Italy; ${ }^{12}$ Interventional Cardiology \\ Unit, San Salvatore Hospital, Pesaro, Italy; ${ }^{13}$ Cardiology and Aortic Centre, The Royal Brompton \& Harefield NHS Trust, London, UK; ${ }^{14}$ Thoracic \\ Aortic Research Center, IRCCS Policlinico San Donato, San Donato, Italy \\ Correspondence to: Marco Di Eusanio. Cardiac Surgery Department, “G. Mazzini” Hospital Piazza Italia, 64100 Teramo, Italy. \\ Email: marco.dieusanio@aslteramo.it.
}

\begin{abstract}
Type A acute aortic dissection (TAAD) is a disease that has a catastrophic impact on a patient's life and emergent surgery represents a key goal of early treatment. Despite continuous improvements in imaging techniques, medical therapy and surgical management, early mortality in patients undergoing TAAD repair still remains high, ranging from $17 \%$ to $26 \%$. In this setting, the International Registry of Acute Aortic Dissection (IRAD), the largest worldwide registry for acute aortic dissection, was established to assess clinical characteristics, management and outcomes of TAAD patients. The present review aimed to evaluate and comment on outcomes of TAAD surgery as reported from IRAD series.
\end{abstract}

Keywords: Type A aortic dissection; aorta; international registry of acute aortic dissection (IRAD); aortic surgery

Submitted May 02, 2016. Accepted for publication May 05, 2016.

doi: 10.21037/acs.2016.05.10

View this article at: http://dx.doi.org/10.21037/acs.2016.05.10

\section{Introduction}

Type A acute aortic dissection (TAAD) is a catastrophic event with a high natural mortality and morbidity (1-3). In recent series, surgical mortality still ranges from $17 \%$ to $26 \%$ and TAAD continues to represent a great challenge for aortic surgeons (4-6). In this setting, the international registry of acute aortic dissection (IRAD), the largest worldwide registry for acute aortic dissection, was established to assess clinical characteristics, management and outcomes of TAAD patients, minimizing the inherent biases seen in small surgical registries or single center series. The present review aimed to evaluate and comment on the outcomes of TAAD surgery, as reported from IRAD analyses.

\section{IRAD design}

The rationale and methodology of the International Registry of Acute Dissection (IRAD) have been published previously $(1,7)$. In brief, IRAD was founded by agreement of 12 aortic centers in 1996, with its main purpose being 
to assess the etiology, clinical features, imaging findings, treatment and outcomes of patients with acute aortic dissection (both type A and type B). Currently, 43 sites enroll patients. Patients with acute aortic dissection were identified either prospectively at presentation or retrospectively by searching hospital discharge diagnosis records and/or the surgery, pathology and imaging databases. The diagnosis of aortic dissection was based on history, imaging studies, direct visualization at surgery and/ or post-mortem findings. Type A acute aortic dissection was defined as any dissection that involved the ascending aorta and/or aortic arch presenting within fourteen days of symptom onset. Patient data were collected using standardized forms, including 290 variables for patient demographics, history, clinical presentation, physical findings, imaging studies, therapeutic management, inhospital mortality, and adverse events. In 2010, IRAD developed the IRAD Interventional Cohort (IRAD-IVC) in order to collect more detailed information (previously not captured in IRAD) on interventional procedures regarding extent of repair, extracorporeal circulation as well as methods of organ and brain protection etc. The completed data forms were forwarded by the participating IRAD sites to the coordinating center at the University of Michigan and reviewed for face validity and completeness.

\section{Demographics}

In a recent IRAD study by Pape et al. assessing outcomes and trends in 4428 consecutive patients with acute aortic dissection enrolled between December 1995 and February 2013, 2,952 (67\%) had type A dissection (8). The mean age of TAAD patients was $62 \pm 14.6$ years. Although less frequently affected by TAAD, women enrolled in IRAD were significantly older than men $(67.9 v s .60 .6$ years) and had a higher mortality than males (33.5\% vs. $24.1 \%)$ (9). A history of hypertension (74.4\%) was the most common predisposing condition and there was also a relatively high proportion of patients who had a history of atherosclerosis $(23.8 \%)$. Furthermore, in the total registry, $4.5 \%$ and $3 \%$ of TAAD were related to Marfan syndrome and iatrogenic causes respectively (8). Several race-related differences have also been described in IRAD patients (10). Among all dissections, TAAD was less frequent in the black population compared with whites ( $47.6 \%$ vs. $60.7 \%)$. Black patients were on average younger $(54.6 \pm 12.8 v s .64 .2 \pm 15.2$ years $)$ and more likely to have a history of cocaine abuse (12\% vs. $1.6 \%)$ and hypertension (89.7\% vs. $73.9 \%)$. Conversely, a history of atherosclerosis, bicuspid aortic valve, existing aortic aneurysm, iatrogenic origin, or prior surgical repair of a dissection or aneurysm were more common in the Caucasian patient cohort.

\section{Clinical presentation}

In IRAD, the most common presenting symptom was the abrupt onset of pain described as 'severe' or the 'worst ever' (93\%), sharp (64.4\%) and frequently localized to the chest $(83 \%)(8)$. One or more pulse deficits was documented in $31 \%$ of patients and hypotension was noted in $25 \%(1,8)$. The latter was often associated with neurologic deficits, myocardial ischemia, mesenteric ischemia or limb ischemia and death. Syncope was reported in $19 \%$ of patients and often indicated the presence of dangerous complications such as cardiac tamponade or obstruction of cerebral vessels $(1,8)$. IRAD data suggest that classic symptoms and signs may be absent in TAAD patients, impeding a prompt establishment of diagnosis. Harris et al. (11) demonstrated that delay in acute aortic dissection diagnosis is more likely in female patients, patients without abrupt onset of pain or those presenting without pain, patients with an absence of pulse deficit or hypotension or patients who initially presented to a non-tertiary care hospital. The registry reported that $6.4 \%$ of patients presented with painless aortic dissection. In these patients hospital mortality was significantly higher than patients with painful dissection (33.3\% vs. 23.2\%) (12). Typical symptoms (abrupt onset of chest or back pain) and signs (aortic regurgitation murmur or pulse deficits) of dissection were less common among the elderly (13). In the 2010 Guidelines on Thoracic Aortic Disease, a risk assessment tool had been proposed in this setting to provide clinicians with a simple and systematic method for rapidly identify patients at high risk and to provide a framework for additional diagnostic testing based on a pretest probability of disease (14). Recently IRAD investigators validated the sensitivity of these risk markers, demonstrating that their application as part of an aortic dissection detection risk score comprise a highly sensitive (96-98\%) clinical tool for the detection of acute aortic dissection (15).

\section{Imaging studies}

Given the frequency of atypical presentations and time dependent mortality, timely and accurate imaging is paramount to the prompt detection and treatment of TAAD. With similar sensitivity, computed tomography 
(CT) and trans-esophageal echocardiography (TEE) have become the standard of care for the diagnosis of this acute aortic syndrome. In IRAD, almost $70 \%$ of patients underwent multiple imaging studies. The first diagnostic modality of choice was CT in $63 \%$, followed by TEE in $32 \%$, aortography in $4 \%$ and MRI in $1 \%$ (16).

\section{Surgical management}

Type A acute aortic dissection is a disease that has a catastrophic impact on a patient's life and emergent surgery represents a key goal of early treatment The mortality of untreated patients with TAAD has been reported to be approximately $1 \%$ to $2 \%$ per hour after symptom onset, with up to $90 \%$ of patients succumbing within 30 days (1-3). The majority of IRAD patients presenting with TAAD were managed surgically ( $86 \%$ overall) with significantly more operative procedures undertaken in the more recent time periods (79\% to $90 \%$ ) (8). The primary aim of surgery is prevention of death from aortic rupture and is mainly accomplished by excision of the proximal intima tear, supracoronary ascending aortic replacement and re-establishment of dominant flow in the distal true lumen (17). Conservative repair of the dissected aortic root with commissural resuspension or prosthetic replacement of the pathologic aortic valve leaflets frequently completes the operation (18). Rampoldi et al. (19) reported on 682 IRAD patients undergoing surgical repair at 18 tertiary centers. A conservative aortic repair (supra-coronary ascending aorta replacement) was performed in $399(58.5 \%)$ patients. More extensive aortic resections involving aortic root replacement were performed in 33.6\% ( $\mathrm{n}=190)$ of patients (aortic valve sparing procedure $5.8 \%$, composite root replacement $16.2 \%)$ and the total arch replacement in $11.5 \%(n=72)$. An open procedure with hypothermic circulatory arrest was used in 597 patients (92\%), with cerebral perfusion in 322 patients (51.4\%). Evidence coming from this study showed that a standardized surgical approach is yet to be defined and that more extensive aortic resection continues to represent a matter of debate. In particular, how to manage the dissected aortic root is still an open question. Although some surgeons advocate a conservative repair of the dissected aortic root involving supracoronary ascending aorta replacement and commissural resuspension or aortic valve replacement to minimize post-operative mortality and morbidity (17), others propose more extensive interventions to replace the entire dissected aortic root tissue $(20,21)$. Compared with conservative root replacement, a more radical root substitution is expected to improve long-term prognosis by minimizing late aortic dilatation, re-dissection and the occurrence of aortic insufficiency. However, this approach is also perceived to carry a higher immediate mortality. Recently IRAD investigators reported on 1995 patients undergoing TAAD surgery, stratifying outcomes according to type of root management: root replacement (RR) $(\mathrm{n}=699,35 \%)$ versus more conservative root management (CR) ( $\mathrm{n}=1,296,65 \%)$. Results demonstrated that a full aortic root replacement did not increase hospital mortality (propensity score-adjusted odds ratio, 1.14) compared to more conservative root treatment. However, midterm survival ( $91.6 \%$ for CR and $92.5 \%$ for RR) and freedom from aortic reoperation were independent of root management type (22).

Therefore the authors concluded that the extension of aortic replacement should remain determined by each patient's clinical and anatomical characteristics as well as by the surgical experience of the operating surgeon. An aggressive approach with replacement of the aortic root seems appropriate in patients with well-acknowledged risk factors for late aortic complications or likely downstream need for re-interventions (young age, connective tissue disease and bicuspid aortic valve).

\section{Results}

In large multicenter surgical registries, in-hospital mortality for TAAD patients ranges from $17 \%$ to $26 \%$ (4-6). In a recent IRAD study assessing trends over a 17 -year span, surgical mortality in patients with TAAD has significantly decreased from $25 \%$ to $18 \%$ (8). Consistent with data reported by others (5,6,23-25), IRAD results suggested that in patients undergoing TAAD surgery, early survival was affected more by preoperative complications and patients' comorbidities than by the type of surgical management $(4,19)$. The principal independent pre-operative predictors of mortality identified in IRAD a were history of aortic valve replacement (OR, 3.12), migrating chest pain (OR, 2.77), pre-operative limb ischemia (OR, 2.10), hypotension during presentation $(\mathrm{OR}, 1.95)$ or shock/cardiac tamponade (OR, 2.69) (4).

Almost one-third of patients presenting with TAAD are over 70 years of age $(26,27)$. In this setting, data from IRAD (26) demonstrated that, although surgical mortality significantly increased with increased age $(38.2 \%$ for patients $\geq 70$ years of age compared to $26.0 \%$ of younger patients), surgical management is still associated with 
significantly lower in-hospital mortality rates compared with optimal medical management $(23.8 \%$ vs. $59.35 \%$ in patients $\leq 80$ years of age). Therefore a surgical approach should be considered in all patients with TAAD and should not be denied on the basis of advanced age alone.

According to risk profile, more than $50 \%$ of IRAD patients who underwent TAAD surgery could be categorized as unstable at the time of surgery. In the registry patients were defined as unstable in the presence of cardiac tamponade, shock, congestive heart failure, cerebrovascular accident, stroke, coma, myocardial ischemia, infarction (or both), acute renal failure, or mesenteric ischemia/infarction at the time of the operation. As would be predicted, inhospital mortality in this group was much higher compared to patients without unstable features (31.4\% vs. $16.7 \%)$ regardless of the type of surgical procedure (4). A predictive factor of survival in this high-risk category of patients was time to operation. Not surprisingly, mean time interval from onset of symptoms to surgical intervention was shorter in unstable patients than in stable patients (3.4vs. 5 hours) (4). Congestive heart failure on presentation was detected in $6.4 \%$ of IRAD patients and was not associated with increased risk mortality (28).

Contrariwise, malperfusion syndromes are associated with elevated mortality and dismal postoperative outcomes in TAAD patients (5,29-31). According to literature, the incidence of brain malperfusion ranges from $7 \%$ to $14 \%$ $(29,30,32,33)$, and significantly affected the therapeutic management. IRAD data (33) showed that nearly 1 out of 10 TAAD patients are complicated by major brain injury at onset of dissection [cerebrovascular accident (CVA) 4.7\%; coma $2.9 \%$ ], carrying a two or threefold higher mortality depending on presence of CVA or coma respectively. Even in this cohort, surgery was found to be a protective factor against hospital mortality in patients with preoperative brain injury (OR, 0.058; $\mathrm{P}<0.001)$, although more than half of these patients do not still undergo TAAD repair. Nevertheless, medical therapy was associated with dismal outcomes: $100 \%$ mortality in patients with coma and $76.2 \%$ in those with CVA. Furthermore, return of brain function was frequent after surgery occurring in $84.3 \%$ of CVA patients and in $78.8 \%$ of those with coma. Therefore TAAD patients with brain injury should always be considered for intervention, especially if they do not present with signs of neurologic devastation.

Mesenteric ischemia is often an insidious end-organ malperfusion complication occurring in $3.7 \%$ of IRAD patients and is an important predictor for hospital mortality
(OR, 2.5) (34). Although mesenteric ischemia is a fairly rare complication, it is very often associated with clinical or imaging signs of other organ injury or malperfusion making the prognosis really unfavorable $(34,35)$. In such patients, when compared with medical therapy, surgery or hybrid management (central aortic operation with percutaneous treatment of mesenteric malperfusion) was associated with better outcomes. Hospital mortality of patients with mesenteric malperfusion receiving medical and surgical/ hybrid therapy was $95.2 \%$ and $41.7 \%$ respectively $(\mathrm{P}<0.001)$. However, IRAD data showed that hybrid management was applied in a very few cases and that immediate surgical repair of the proximal dissected aorta still represents the most common therapeutic approach for patients with TAAD complicated by mesenteric malperfusion (34).

Pulse deficits are common signs in TAAD patients and were associated with higher risk of early mortality and postoperative complications (RR, 2.73) (36). Pre-operative limb ischemia was reported in $9.7 \%$ of IRAD cohort and emerged as independent predictor (OR, 2.1) of subsequent operative mortality (4)

As shown by IRAD data, the aforementioned TAADrelated complications increased operative mortality and morbidity in patients undergoing TAAD repair. Nevertheless, surgery was consistently associated with considerably lower in-hospital mortality rates compared with medical treatment. Therefore surgery should be considered in all TAAD patients, but the definitive management should be individualized for each case based on patient clinical and anatomical characteristics, institutional volume of TAAD operations, surgical experience of the operating surgeon and patient/family preferences $(6,37)$.

\section{Conclusions}

Data from IRAD showed that mortality in TAAD patients remains high even though surgical outcomes have significantly improved over the past 20 years. Prognosis of TAAD patients was largely determined by patient's characteristics, comorbidities and dissection-related complications; in particular surgical mortality was mainly related to unstable patients with pre-operative organ malperfusion. Compared with other forms of therapy, surgery was associated with superior survival, even in highrisk patients, and thus should be considered in all patients with definitive management individualized for each case based on patients' characteristics and surgical experience of the operating surgeons. 


\section{Acknowledgements}

This research was generously supported by W. L. Gore \& Associates, Inc., Medtronic, the Varbedian Aortic Research Fund, the Hewlett Foundation, the Mardigian Foundation, UM Faculty Group Practice, Terumo, and Ann and Bob Aikens.

\section{Footnote}

Conflicts of Interest: The authors have no conflicts of interest to declare.

\section{References}

1. Hagan PG, Nienaber CA, Isselbacher EM, et al. The International Registry of Acute Aortic Dissection (IRAD): new insights into an old disease. JAMA 2000;283:897-903.

2. Fann JI, Smith JA, Miller DC, et al. Surgical management of aortic dissection during a 30-year period. Circulation 1995;92:II113-21.

3. Scholl FG, Coady MA, Davies R, et al. Interval or permanent nonoperative management of acute type A aortic dissection. Arch Surg 1999;134:402-5; discussion 405-6.

4. Trimarchi S, Nienaber CA, Rampoldi V, et al. Contemporary results of surgery in acute type A aortic dissection: The International Registry of Acute Aortic Dissection experience. J Thorac Cardiovasc Surg 2005;129:112-22.

5. Conzelmann LO, Weigang E, Mehlhorn U, et al. Mortality in patients with acute aortic dissection type A: analysis of pre- and intraoperative risk factors from the German Registry for Acute Aortic Dissection Type A (GERAADA). Eur J Cardiothorac Surg 2016;49:e44-52.

6. Knipp BS, Deeb GM, Prager RL, et al. A contemporary analysis of outcomes for operative repair of type A aortic dissection in the United States. Surgery 2007;142:524-8; discussion 528.

7. Tsai TT, Trimarchi S, Nienaber CA. Acute aortic dissection: perspectives from the International Registry of Acute Aortic Dissection (IRAD). Eur J Vasc Endovasc Surg 2009;37:149-59.

8. Pape LA, Awais M, Woznicki EM, et al. Presentation, Diagnosis, and Outcomes of Acute Aortic Dissection: 17Year Trends From the International Registry of Acute Aortic Dissection. J Am Coll Cardiol 2015;66:350-8.

9. Nienaber CA, Fattori R, Mehta RH, et al. Gender- related differences in acute aortic dissection. Circulation 2004;109:3014-21.

10. Bossone E, Pyeritz RE, O'Gara P, et al. Acute aortic dissection in blacks: insights from the International Registry of Acute Aortic Dissection. Am J Med 2013;126:909-15.

11. Harris KM, Strauss CE, Eagle KA, et al. Correlates of delayed recognition and treatment of acute type A aortic dissection: the International Registry of Acute Aortic Dissection (IRAD). Circulation 2011;124:1911-8.

12. Park SW, Hutchison S, Mehta RH, et al. Association of painless acute aortic dissection with increased mortality. Mayo Clin Proc 2004;79:1252-7.

13. Mehta RH, O'Gara PT, Bossone E, et al. Acute type A aortic dissection in the elderly: clinical characteristics, management, and outcomes in the current era. J Am Coll Cardiol 2002;40:685-92.

14. Hiratzka LF, Bakris GL, Beckman JA, et al. 2010 ACCF/AHA/AATS/ACR/ASA/SCA/SCAI/SIR/STS/ SVM guidelines for the diagnosis and management of patients with Thoracic Aortic Disease: a report of the American College of Cardiology Foundation/American Heart Association Task Force on Practice Guidelines, American Association for Thoracic Surgery, American College of Radiology, American Stroke Association, Society of Cardiovascular Anesthesiologists, Society for Cardiovascular Angiography and Interventions, Society of Interventional Radiology, Society of Thoracic Surgeons, and Society for Vascular Medicine. Circulation 2010;121:e266-369.

15. Rogers AM, Hermann LK, Booher AM, et al. Sensitivity of the aortic dissection detection risk score, a novel guidelinebased tool for identification of acute aortic dissection at initial presentation: results from the international registry of acute aortic dissection. Circulation 2011;123:2213-8.

16. Moore AG, Eagle KA, Bruckman D, et al. Choice of computed tomography, transesophageal echocardiography, magnetic resonance imaging, and aortography in acute aortic dissection: International Registry of Acute Aortic Dissection (IRAD). Am J Cardiol 2002;89:1235-8.

17. Westaby S, Saito S, Katsumata T. Acute type A dissection: conservative methods provide consistently low mortality. Ann Thorac Surg 2002;73:707-13.

18. Geirsson A, Bavaria JE, Swarr D, et al. Fate of the residual distal and proximal aorta after acute type a dissection repair using a contemporary surgical reconstruction algorithm. Ann Thorac Surg 2007;84:1955-64; discussion 1955-64. 
19. Rampoldi V, Trimarchi S, Eagle KA, et al. Simple risk models to predict surgical mortality in acute type A aortic dissection: the International Registry of Acute Aortic Dissection score. Ann Thorac Surg 2007;83:55-61.

20. Halstead JC, Spielvogel D, Meier DM, et al. Composite aortic root replacement in acute type A dissection: time to rethink the indications? Eur J Cardiothorac Surg 2005;27:626-32; discussion 632-3.

21. Kallenbach K, Oelze T, Salcher R, et al. Evolving strategies for treatment of acute aortic dissection type A. Circulation 2004;110:II243-9.

22. Di Eusanio M, Trimarchi S, Peterson MD, et al. Root replacement surgery versus more conservative management during type A acute aortic dissection repair. Ann Thorac Surg 2014;98:2078-84.

23. Kazui T, Washiyama N, Bashar AH, et al. Surgical outcome of acute type A aortic dissection: analysis of risk factors. Ann Thorac Surg 2002;74:75-81; discussion 81-2.

24. Ehrlich MP, Ergin MA, McCullough JN, et al. Results of immediate surgical treatment of all acute type A dissections. Circulation 2000;102:III248-52.

25. Goda M, Imoto K, Suzuki S, et al. Risk analysis for hospital mortality in patients with acute type a aortic dissection. Ann Thorac Surg 2010;90:1246-50.

26. Trimarchi S, Eagle KA, Nienaber CA, et al. Role of age in acute type A aortic dissection outcome: report from the International Registry of Acute Aortic Dissection (IRAD). J Thorac Cardiovasc Surg 2010;140:784-9.

27. Rylski B, Suedkamp M, Beyersdorf F, et al. Outcome after surgery for acute aortic dissection type A in patients over 70 years: data analysis from the German Registry for Acute Aortic Dissection Type A (GERAADA). Eur J Cardiothorac Surg 2011;40:435-40.

28. Tsai TT, Bossone E, Isselbacher EM, et al. Clinical characteristics of hypotension in patients with acute aortic dissection. Am J Cardiol 2005;95:48-52.

Cite this article as: Berretta P, Patel HJ, Gleason TG, Sundt TM, Myrmel T, Desai N, Korach A, Panza A, Bavaria J, Khoynezhad A, Woznicki E, Montgomery D, Isselbacher EM, Di Bartolomeo R, Fattori R, Nienaber CA, Eagle KA, Trimarchi S, Di Eusanio M. IRAD experience on surgical type A acute dissection patients: results and predictors of mortality. Ann Cardiothorac Surg 2016;5(4):346-351. doi: 10.21037/ acs.2016.05.10
29. Geirsson A, Szeto WY, Pochettino A, et al. Significance of malperfusion syndromes prior to contemporary surgical repair for acute type A dissection: outcomes and need for additional revascularizations. Eur J Cardiothorac Surg 2007;32:255-62.

30. Girdauskas E, Kuntze T, Borger MA, et al. Surgical risk of preoperative malperfusion in acute type A aortic dissection. J Thorac Cardiovasc Surg 2009;138:1363-9.

31. Augoustides JG, Szeto WY, Desai ND, et al. Classification of acute type A dissection: focus on clinical presentation and extent. Eur J Cardiothorac Surg 2011;39:519-22.

32. Immer FF, Grobéty V, Lauten A, et al. Does malperfusion syndrome affect early and mid-term outcome in patients suffering from acute type A aortic dissection? Interact Cardiovasc Thorac Surg 2006;5:187-90.

33. Di Eusanio M, Patel HJ, Nienaber CA, et al. Patients with type A acute aortic dissection presenting with major brain injury: should we operate on them? J Thorac Cardiovasc Surg 2013;145:S213-21.

34. Di Eusanio M, Trimarchi S, Patel HJ, et al. Clinical presentation, management, and short-term outcome of patients with type A acute dissection complicated by mesenteric malperfusion: observations from the International Registry of Acute Aortic Dissection. J Thorac Cardiovasc Surg 2013;145:385-90.

35. Pacini D, Leone A, Belotti LM, et al. Acute type A aortic dissection: significance of multiorgan malperfusion. Eur J Cardiothorac Surg 2013;43:820-6.

36. Bossone E, Rampoldi V, Nienaber CA, et al. Usefulness of pulse deficit to predict in-hospital complications and mortality in patients with acute type A aortic dissection. Am J Cardiol 2002;89:851-5.

37. Iribarne A, Milner R, Merlo AE, et al. Outcomes following emergent open repair for thoracic aortic dissection are improved at higher volume centers. J Card Surg 2015;30:74-9. 